\title{
On the relevance of bubbles and potential flows for stellar convection
}

\author{
M. M. Miller Bertolami, ${ }^{1,2 \star}$ M. Viallet, ${ }^{1}$ V. Prat, ${ }^{1}$ W. Barsukow ${ }^{3}$ and A. Weiss ${ }^{1}$ \\ ${ }^{1}$ Max-Planck-Institut für Astrophysik, Karl-Schwarzschild-Str. 1, D-85748 Garching, Germany \\ ${ }^{2}$ Instituto de Astrofísica de La Plata, UNLP-CONICET, Paseo del Bosque s/n, 1900 La Plata, Argentina \\ ${ }^{3}$ Institut für Mathematik, Universität Würzburg, Campus Hubland Nord, Emil-Fischer-Strasse 40, D-97074 Würzburg, Germany
}

Accepted 2016 January 20. Received 2016 January 20; in original form 2015 November 6

\begin{abstract}
Recently Pasetto et al. have proposed a new method to derive a convection theory appropriate for the implementation in stellar evolution codes. Their approach is based on the simple physical picture of spherical bubbles moving within a potential flow in dynamically unstable regions, and a detailed computation of the bubble dynamics. Based on this approach, the authors derive a new theory of convection which is claimed to be parameter-free, non-local and time-dependent. This is a very strong claim, as such a theory is the holy grail of stellar physics. Unfortunately, we have identified several distinct problems in the derivation which ultimately render their theory inapplicable to any physical regime. In addition, we show that the framework of spherical bubbles in potential flows is unable to capture the essence of stellar convection, even when equations are derived correctly.
\end{abstract}

Key words: convection-stars: evolution-stars: fundamental parameters.

\section{INTRODUCTION}

It is not an exaggeration to state that the turbulent transport of heat, angular momentum and chemical species is the most important unsolved problem in stellar astrophysics. Most of the present uncertainties in stellar physics are, in one way or another, linked to our incomplete understanding of mixing in stellar interiors, e.g. the final fate of stars of high and intermediate mass, formation of s-process elements, chemical anomalies on the red giant branch, formation of carbon stars, size of the convective cores in $\mathrm{H}$ - and He-burning stars. In spite of many decades of attempts to derive an accurate time-dependent and non-local theory of convection that can be included in stellar evolution codes, success has been very minor. While some theories of time-dependent convection have been derived and applied (Kuhfuss 1986; Kuhfuss 1987; Wuchterl \& Feuchtinger 1998; Flaskamp 2003), they all introduce several free parameters that must be calibrated for different regimes, diminishing their predictive power. Even more problematic is the case of non-local convection and convective boundary mixing. For decades, serious attempts have been made to derive non-local convection theories that could be introduced in stellar evolution codes (Deng, Xiong \& Chan 2006; Deng \& Xiong 2008; Canuto 2011e). However, these theories are not popular due to their complexity and their limited accuracy (Xiong 1986; Weiss \& Flaskamp 2007). Attempts to derive a general framework for the treatment of stellar convection and other mixing processes lead to very complex equations which cannot be easily included in 1D stellar evolution codes (Kuhfuss 1986; Canuto 2011a,b,c,d,e). In fact, non-local consequences of convection, such as convective boundary mixing, are routinely included in stellar evolution codes based on ad hoc prescriptions and additional free parameters - see Viallet et al. (2015) and Arnett et al. (2015), for recent discussions on these issues. Consequently, despite its well-known shortcomings, the mixing-length theory (MLT; Prandtl 1925; Biermann 1932; Vitense 1953) has been in use for more than 80 years.

In this paper, we call a 'theory of stellar convection' a theory that can be implemented in 1D stellar evolution codes. Such a theory should capture the essential properties of turbulent convection, allowing us to reproduce its effects on the stellar structure (mainly chemical mixing and energy transport) without the need to resort to expensive 3D simulations. A theory of stellar convection is highly sought, as the predictive power of current stellar models is strongly limited by the shortcomings of MLT. The status on the theoretical side contrasts with the progress done in observational techniques and instrumentation (e.g. Kepler, CoRoT and Gaia; de Bruijne 2012; Guzik et al. 2014). A new generation of stellar models is necessary to fully exploit the large amount of quality data that is delivered by observers. Without any doubt, a new generation of stellar models should rely on a better treatment of convection.

Recently, Pasetto et al. (2014) claimed to present an accurate parameter-free, non-local, time-dependent theory of stellar convection that can be easily implemented in 1D stellar codes. This is a strong claim since such a theory has been sought for many decades. In order to facilitate the reader's understanding, we start by schematically summarizing the method proposed by Pasetto et al. (2014). Pasetto et al. (2014) adopt a rather simple picture of convection, in which the transport of heat is achieved by 'bubbles' that rise 
due to buoyancy in a convectively unstable region. This description of convection using the concept of bubbles is likely inspired from the usual simple picture that one has in mind when deriving the MLT. Furthermore, in the picture of Pasetto et al. (2014), convective bubbles have a definite shape (they are spherical) and are differentiated from the surrounding material - i.e. the surrounding material flows around them. As a first step, the authors analyse the motion of an isolated bubble. From kinematic considerations, they derive the expression of the velocity field around the bubble. This is done assuming that the flow around the bubble is a potential flow ( $\nabla \times \boldsymbol{u}=0$, where $\boldsymbol{u}$ is the velocity field). This allows them to link the velocity of the moving bubble to that of the surrounding fluid at each time - i.e. assuming an instantaneous adjustment of the surrounding fluid. Given the velocity field around the bubble, the authors then deduce the pressure field around the bubble. Knowing the pressure field, they compute the total force that the fluid exerts on the bubble,

$\vec{F}=\oint_{\text {bubble }} P \boldsymbol{n} \mathrm{d} S$.

Applying Newton's law to the bubble, the authors derive an expression for the acceleration of the bubble, the first key result of their theory. With appropriate initial conditions, this equation defines completely the motion of the bubble as a function of time. In the second part of their work, Pasetto et al. (2014) use their theory for an isolated bubble to formulate a theory of convection by considering a collective set of bubbles.

In this work, we study the applicability of this method to the stellar regime and its possible limitations. In order to do this, we derive the equation for the dynamics of the bubble by a careful accounting of the physical assumptions and hypothesis made in the derivation. During this process, we found that some inconsistent physical and mathematical assumptions have been made by Pasetto et al. (2014), casting serious doubts on the validity of their theory. It will also become clear in the next section that the claim of a non-local, time-dependent theory is an overstatement by the authors. Yet, the method of deriving a parameter-free convection theory from the full dynamics of a convective element assuming a surrounding potential flow is interesting. If valid, the method could indeed be extended to obtain a parameter-free, non-local and time-dependent theory and to get rid of the mixing length parameter, $\alpha$, whose calibration in different stellar regimes is problematic (Ludwig, Freytag \& Steffen 1999; Trampedach et al. 2014; Magic, Weiss \& Asplund 2015; Tremblay et al. 2015). We have been able to reobtain the dynamics of the bubble by a sound mathematical and physical derivation. This allows us to study the behaviour of the solutions and assess the physical regime in which the method described by Pasetto et al. (2014) can be applied. Unfortunately, we find that the movement of spherical bubbles within potential flows is completely inapplicable to the regime of stellar convection and that no useful theory of stellar convection can be obtained from this approach. This is not a surprise since the adoption of the ideal fluid and the potential flow approximations (the 'dry water' approximation; Feynman 1964) neglect the importance of viscosity and boundary layers for the dynamics of the bubble.

The paper is organized as follows. In the next section, we show that the derivation of Pasetto et al. (2014) of the acceleration equation is flawed due to incorrect physical and mathematical assumptions. In Section 3, we clarify the approximations underlying their theory of an isolated bubble, and provide the correct derivation of the acceleration equation of the bubble. We show that the authors misinterpreted their acceleration equation, and neglected a term that is physically important. In Section 4, we provide the correct analysis of the equation of motion, and focus particularly on the asymptotic/final regimes reached by the bubble. We show that it is unavoidable that the theory becomes inconsistent and highly nonphysical. In Section 5, we finish the article with some discussion and concluding remarks.

\section{PHYSICAL AND MATHEMATICAL INCONSISTENCIES IN PASETTO ET AL. (2014)}

Before analysing the physical and mathematical assumptions adopted by Pasetto et al. (2014), it is already worth noting that a first consequence of the method adopted by the authors is that it cannot provide a self-consistent time-dependent and non-local convection theory in the usual sense of these terms. By looking at the system of equations that define the theory of convection presented in Pasetto et al. (2014), see their equation $60^{1}$, it becomes apparent that their theory is a local formulation, very much in the spirit of MLT. In a local theory of convection, velocities and convective fluxes depend only on the local thermodynamical variables and their local gradients. Usually, a local theory of convection results from a 'local' approach to the problem of convection. In a local approach, one makes the assumption that all the relevant processes are taking place on length-scales $l$ that are much smaller than the typical length-scale over which the background is changing, i.e. $l \ll H_{\mathrm{p}}$, $H_{\rho}$, where $l$ is the length-scale of the process of interest, $H_{\mathrm{p}}$ and $H_{\rho}$ are the pressure and density scaleheight, respectively. Clearly, the work presented in Pasetto et al. (2014) follows such a local approach, as clearly stated in their Section $2 .^{2}$ It is not possible to derive a self-consistent non-local theory of convection from such a local approach, as it is precisely the local approach that decouples the problem at each radius. Furthermore, a 'time-dependent' theory of convection has a very specific meaning in the field of 1D stellar structure computations. It refers to a theory which is able to describe convection in the case where the stellar background evolves on a time-scale smaller, or of the same order, than the convective turn-over time-scale. As mentioned in the introduction, such theories exist but their predictive power is hampered by several free parameters. As admitted by the authors in one of their footnotes, the theory presented in Pasetto et al. (2014) is not 'time-dependent' in the usual sense. ${ }^{3}$ Very likely, Pasetto et al. (2014) refer to their

\footnotetext{
${ }^{1}$ Throughout this paper, we denote the equations in Pasetto et al. (2014) with square brackets to differentiate them from our own equations.

${ }^{2}$ Where they state, 'We proceed furthermore with an additional simplification by assuming that the stellar fluid is incompressible and irrotational on large distance scales. The concept of a large distance scale for incompressibility and irrotationality is defined here from a heuristic point of view: this length should be large enough to contain a significant number of convective elements so that a statistical formulation is possible when describing the mean convective flux of energy, but small enough so that the distance travelled by the convective element is short compared to the typical distance over which significant gradients in temperature, density, pressure, etc. can develop (i.e. those gradients are locally small).'

3 This is hinted by the authors at the end of section 2, p. 3594; 'Before starting our analysis, in order to avoid a possible misunderstanding of the real meaning of some of our analytical results, it might be wise to call attention to a formal aspect of the mathematical notation we have adopted. For some quantities, $Q$ function of time or space or both, $Q(x ; t)$, we look at their asymptotic behaviour by formally taking the limits $Q^{\infty}=\lim _{x \rightarrow x^{\infty}, t \rightarrow \infty} Q(x ; t)$. This does not mean that we are taking temporal intervals infinitely long, rather that we are considering time long enough so that the asymptotic trend of the quantity $\mathrm{Q}$ is reached but still short enough so that the physical properties
} 
theory as being 'time-dependent' because they integrate in time a set of equations until an asymptotic regime is obtained. However, their theory of convection is based on the asymptotic regime, where the time variable is not relevant any more and, consequently it cannot be considered as a theory of time-dependent convection.

Having clarified that the approach derived by Pasetto et al. (2014) deals with a time-independent and local theory, we now turn to analyse some of the mathematical and physical approximations made in their derivation of the equation of motion for the spherical bubble.

\subsection{The physical assumptions}

After deriving the equations for the velocity field of an incompressible and irrotational fluid around an expanding sphere moving within a fluid of constant density and in hydrostatic equilibrium at infinity (see their sections 2 and 3), the authors apply this result to compute the forces exerted on the sphere by the surrounding fluid. Besides the assumptions of an incompressible and irrotational fluid of constant density, they also neglect heat diffusion and restrict themselves to the subsonic regime (i.e. spheres moving at speeds much smaller than the speed of sound). In this context, they claim that it is reasonable to assume that (see their equation 12)

$\frac{v_{\mathrm{b}}}{\dot{R}} \ll 1, \forall t>t_{\min }$,

i.e. that the relative velocity $v_{\mathrm{b}}=\left|\boldsymbol{v}_{\boldsymbol{b}}\right|$ between the convective element and the intrastellar medium is much smaller than its expansion velocity $\dot{R}=|\dot{\boldsymbol{R}}|-$ throughout this work, we denote the radius of the bubble by $R$, and its temporal changes by $\dot{R}$ and $\ddot{R}$. However, it is easy to show that such a regime is in strong contradiction with the assumptions of a subsonic regime and a local approach - the latter materialized by the possibility of solving the movement of the bubble assuming a medium of constant density.

Let us say that a bubble is characterized by its mass $m_{\mathrm{b}}$ (constant in time), density $\rho_{\mathrm{b}}(t)$, pressure $P_{\mathrm{b}}(t)$, radius $R(t)$, position $r_{\mathrm{b}}(t)$, and velocity $v_{\mathrm{b}}(t)=\dot{r}_{\mathrm{b}}$. The surrounding medium is characterized by its pressure stratification $P(r)$. First, a spherical bubble travelling in the surrounding medium at a subsonic speed remains in pressure equilibrium, i.e. $P_{\mathrm{b}} \simeq P$ as sound waves are able to wash out any pressure difference. ${ }^{4}$ Therefore, $P_{\mathrm{b}}(t)=P\left(r_{\mathrm{b}}(t)\right)$ and, taking the time derivative, one obtains

$\frac{\mathrm{d} P_{\mathrm{b}}(t)}{\mathrm{d} t}=\frac{\mathrm{d} P\left(r_{\mathrm{b}}(t)\right)}{\mathrm{d} t}=\frac{\mathrm{d} P}{\mathrm{~d} r} v_{\mathrm{b}}=-\frac{P v_{\mathrm{b}}}{H_{\mathrm{p}}}$,

or simply

$\frac{\mathrm{d} \log P_{\mathrm{b}}}{\mathrm{d} t}=-\frac{v_{\mathrm{b}}}{H_{\mathrm{p}}}$

We used the definition of the pressure scaleheight $H_{\mathrm{p}}=-\frac{\mathrm{d} r}{\mathrm{~d} \log P}$. Neglecting heat conduction, the change in density of the bubble follows the adiabatic relation

$\frac{P_{\mathrm{b}}}{\rho_{\mathrm{b}} \Gamma_{1}}=$ const,

of the whole system have not changed significantly, such as that the star still exists.'

${ }^{4}$ In addition, we show in Appendix A that this is also mathematically consistent with the equations for the dynamics of the bubble to be derived later. where $\Gamma_{1}$ is the first adiabatic index. This is equivalent to

$\frac{\mathrm{d} \log P_{\mathrm{b}}}{\mathrm{d} t}=\Gamma_{1} \frac{\mathrm{d} \log \rho_{\mathrm{b}}}{\mathrm{dt}}$.

Combining equations (3) and (5), we obtain:

$\Gamma_{1} \frac{\mathrm{d} \log \rho_{\mathrm{b}}}{\mathrm{d} t}=-\frac{v_{\mathrm{b}}}{H_{\mathrm{p}}}$.

Finally, as the mass of the bubble is constant, its density decreases as $\rho_{\mathrm{b}} \propto R^{-3}$. Thus, we obtain:

$-3 \Gamma_{1} \frac{\mathrm{d} \log R}{\mathrm{~d} t}=-\frac{v_{\mathrm{b}}}{H_{\mathrm{p}}}$.

It follows that, within the adiabatic and subsonic approximations, the relation between the expansion rate and the velocity of the bubble is

$\frac{v_{\mathrm{b}}}{\dot{R}}=\frac{3 H_{\mathrm{p}} \Gamma_{1}}{R}$.

We can conclude that the assumption $v_{\mathrm{b}} / \dot{R} \ll 1$ is equivalent to $H_{\mathrm{p}} / R \ll 1$, as usually $\Gamma_{1} \sim 1$.

This result can be understood on the basis of the following very simple physical observation. Within the subsonic approximation, the only way in which a bubble can expand much faster than it moves is when small vertical displacements lead to big changes in the pressure of the surrounding fluid, i.e. when $H_{\mathrm{p}}$ is very small compared to the size of the bubble.

Unfortunately, assuming $v_{\mathrm{b}} / \dot{R} \ll 1$, which implies $H_{\mathrm{p}} / R \ll 1$, is in complete contradiction with the core of the theory which is based on a local picture of convection. In particular it is in clear contradiction with expressions such as equations 3, 13, 24 and 27 from Pasetto et al. (2014) which are derived within the picture of a bubble moving in a constant density background.

\subsection{The mathematical approximations}

While the previous inconsistency is serious enough to render the applicability of the theory questionable, other contradictions develop as a consequence of mathematical simplifications during the derivation of the force exerted by the fluid over the moving sphere - sections 4.2 and 5 of Pasetto et al. (2014). The first of these approximations comes during the derivation of 'Lemma 1' of Pasetto et al. (2014) (equation 13). There it is stated that, under the validity of $v_{\mathrm{b}} / \dot{R} \ll 1$, it is possible to say that

$$
\left(\frac{v_{\mathrm{b}}}{\dot{R}}\right)^{2} \frac{1}{2}\left(\frac{9}{4} \sin ^{2} \theta-1\right) \ll \dot{v}_{\mathrm{b}} \frac{R}{\dot{R}^{2}}\left(\frac{3}{2} \cos \theta-\cos \phi\right)+\frac{\ddot{R} R}{\dot{R}^{2}},
$$

and also that

$$
\left(\frac{v_{\mathrm{b}} \dot{R}}{\dot{R}^{2}}\right)^{2} \frac{5}{2} \cos \theta \ll \dot{v}_{\mathrm{b}} \frac{R}{\dot{R}^{2}}\left(\frac{3}{2} \cos \theta-\cos \phi\right)+\frac{\ddot{R} R}{\dot{R}^{2}} .
$$

It is clear that it is not possible to justify these two inequalities (equation 14 in Pasetto et al. 2014) solely on the base of $v_{\mathrm{b}} / \dot{R} \ll 1$ without any other assumption. In order to justify equations (9) and (10), one must make the assumptions that $v_{\mathrm{b}}^{2} \ll\left|\dot{v}_{\mathrm{b}} R\right|$ and $v_{\mathrm{b}}^{2} \ll$ $|\ddot{R} R|$. These two assumptions restrict even more the physical regime in which the theory could be applicable. One might wonder whether such specific regime, i.e. $v_{\mathrm{b}} / \dot{R} \ll 1, v_{\mathrm{b}}^{2} \ll\left|\dot{v}_{\mathrm{b}} R\right|$ and $v_{\mathrm{b}}^{2} \ll|\ddot{R} R|$, does exist at all.

We will show later that the two incorrect approximations performed in equations (9) and (10) do not change the shape of the 
equation for the acceleration of the fluid element, although they do change some of the coefficients. Unfortunately, after the derivation the equation of motion (their equation 24$)^{5}$

$\dot{\boldsymbol{v}}_{\boldsymbol{b}}=\boldsymbol{g} \frac{m_{\mathrm{b}}-M}{m_{\mathrm{b}}+M / 2}-\frac{10}{3} \frac{\pi R^{2} \rho \boldsymbol{v}_{\mathrm{b}} \dot{R}}{m_{\mathrm{b}}+M / 2}$,

the authors simplify this expression by neglecting the second term to obtain their equation 26 . It is not possible to neglect the second term solely on the base of $v_{\mathrm{b}} / \dot{R} \ll 1$, as it is claimed by Pasetto et al. (2014). The physical regime in which this term can be neglected is discussed below. It is worth noting that their equation 26 plays a key role in the derivation of the convective theory, as it is equation 26 that is used in the further development of the work - e.g. in the derivation of their equation 27. Interestingly, by doing this, the authors dropped the only term that could provide them with a truly asymptotic regime, as we will show in Section 4. It is easy to see that, the actual physical regime in which the second term becomes negligible is the one of strong buoyancy forces $\left(M-m_{\mathrm{b}}\right) / m_{\mathrm{b}} \sim 1$. A simple rewriting of their equation 24 using the definition of $M=4 \pi R^{3} \rho / 3$, shows that

$\dot{\boldsymbol{v}}_{\mathrm{b}}=\boldsymbol{g} \frac{m_{\mathrm{b}}-M}{m_{\mathrm{b}}+M / 2}-\frac{10}{4} \frac{M \boldsymbol{v}_{\mathrm{b}}(\dot{R} / R)}{m_{\mathrm{b}}+M / 2}$.

It follows that, for strong buoyancy forces, the second term becomes negligible when $g \gg v_{\mathrm{b}} \dot{R} / R$. Using that $H_{\mathrm{p}}=P / g \rho$ and that for an ideal gas, the sound speed is $c_{\mathrm{s}}{ }^{2}=\gamma P / \rho$, we see that the second term becomes negligible if $c_{\mathrm{s}}{ }^{2} / \gamma H_{\mathrm{p}} \gg \dot{R} / R v_{\mathrm{b}}$. As the derivation of the equation of motion within a local picture requires $H_{\mathrm{p}} \gg R$, the previous condition holds as soon as $c_{\mathrm{s}}{ }^{2} / \gamma \gg \dot{R} v_{\mathrm{b}}$. As a result, we see that the second term is indeed negligible as soon as we have significant buoyancy forces $\left(M-m_{\mathrm{b}}\right) / m_{\mathrm{b}} \gtrsim 1$ and we restrain ourselves to subsonic motions and expansions. The previous argument shows that, although for very different reasons, in the regime of significant buoyancy and subsonic bubbles, the key equation 26 of Pasetto et al. (2014) is valid.

Finally, a serious inconsistency arises during their computation of the convective flux in their section 6 . In order to compute the velocity of the convective elements (their equation 41), the authors analyse the movement of the stagnation points in the case of a nonexpanding rigid-body movement $(\dot{R}=\ddot{R}=0)$. The approximation of a non-expanding convective element is in stark contradiction with the previous derivation of theory. Furthermore, the authors wrongly assume that $P / \rho+\Phi_{\mathrm{g}} \simeq 0$ at the stagnation points. From this analysis, Pasetto et al. (2014) conclude that the velocity, radius and acceleration of the bubble are connected by (see their equation 41)

$v_{\mathrm{b}}^{2}=-\dot{v}_{\mathrm{b}} R$

Clearly, assuming equation (13) is in apparent contradiction with equations (9) and (10), which require $v_{\mathrm{b}}^{2} \ll\left|\dot{v}_{\mathrm{b}} R\right|$. The neglection of the second term in equation (12) is also in contradiction with the simultaneous assumption of $v_{\mathrm{b}}^{2}=-\dot{v}_{\mathrm{b}} R$ and equation (1) - as these assumptions imply $v_{\mathrm{b}} \dot{R} / R \gg \dot{v}_{\mathrm{b}}$. We will show in Section 4 that the ratio $v_{\mathrm{b}}^{2} /\left(\dot{v}_{\mathrm{b}} R\right)$ changes by orders of magnitude during the actual motion of the bubble (see Figs 1 and 2). Consequently, equation (13) does not hold.

\footnotetext{
${ }^{5}$ We have corrected the sign of the first term, because when $M>m_{\mathrm{b}}$ (more buoyancy than weight) the direction of $\dot{\boldsymbol{v}}_{\mathbf{b}}$ should be opposite to that of $\boldsymbol{g}$ and, also, have added the denominator of the first term $\left(m_{\mathrm{b}}+M / 2\right)$ which should also appear in the second term.
}

\section{EQUATION OF MOTION FOR AN EXPANDING SPHERE IN A POTENTIAL FLOW}

As mentioned during the introduction, during the study of Pasetto et al. (2014) we found that the equivalent of their key equation 24 (equation 11) can be derived in a sound physical and mathematical way. This is an interesting result which will allow us to study the motion of an isolated bubble within the present picture and assess its applicability to derive a theory of stellar convection.

In line with Pasetto et al. (2014), we will assume that the fluid is ideal (no viscosity), incompressible $(\nabla \cdot v=0)$ and irrotational $(\nabla \times v=0)$. We will assume that the path travelled by the sphere $\left(l_{\mathrm{b}}\right)$ can be considered small compared to the distances over which pressure $P$, gravity $\boldsymbol{g}$ or density $\rho$ change. If $H_{\mathrm{p}}$ and $H_{\rho}$ are the pressure and density scaleheights, we have $l_{\mathrm{b}} \ll H_{\mathrm{p}}$ and $l_{\mathrm{b}} \ll H_{\rho}$. The medium is assumed to be in hydrostatic equilibrium far from the moving element $\left(\nabla P_{\infty}=\rho g\right.$; where $P_{\infty}$ means the pressure in that layer and far away from the bubble).

\subsection{Flow around an expanding sphere moving at constant velocity}

Under the assumption $\nabla \times \boldsymbol{v}=0$, there is a potential $\psi$ so that $\nabla \psi=\boldsymbol{v}$. The potential of an incompressible flow of constant density must fulfill $\nabla^{2} \psi=0$ - see section 9 of Landau \& Lifshitz (1987), for a detailed discussion of potential flows. In particular, the solution corresponding to the motion (with velocity $\boldsymbol{v}_{\mathrm{b}}=v_{\mathrm{b}} \boldsymbol{e}_{\mathrm{z}}$ ) of an expanding sphere (of radius $R$ and expansion rate $\dot{R}$ ) within a fluid which is in hydrostatic equilibrium far away (i.e. $|\boldsymbol{x}| \rightarrow \infty$ ) can be obtained by solving

$\nabla^{2} \psi=0$

with the boundary conditions

$$
\begin{aligned}
& \forall t, \lim _{|x| \rightarrow \infty} \boldsymbol{v}=0, \\
& \forall t, \forall \boldsymbol{n}^{\prime}, \boldsymbol{v} \cdot \boldsymbol{n}^{\prime}=\dot{R}+\boldsymbol{v}_{b} \cdot \boldsymbol{n}^{\prime}, \\
& \text { on the sphere }\left|\boldsymbol{x}-\boldsymbol{r}_{b}\right|=R \text {, }
\end{aligned}
$$

where we denote the position of the bubble by $\boldsymbol{r}_{\mathbf{b}}(t)$ and we define $n^{\prime}=x^{\prime} /\left|x^{\prime}\right|$, with $x^{\prime}=\boldsymbol{x}-r_{\mathrm{b}}$ the position as seen from the centre of the bubble.

It is easier to solve the problem by changing to the coordinate system comoving with the sphere at constant velocity $\boldsymbol{v}_{\mathrm{b}}$. From that coordinate system, the problem reduces to that of an expanding sphere at rest located at $\boldsymbol{x}^{\prime}=0$ within a fluid moving at infinity with $v_{\infty}=-v_{\mathrm{b}}$, i.e. to solving

$\nabla^{\prime 2} \varphi=0$,

where $\nabla^{\prime}$ denotes the derivatives with respect to $\boldsymbol{x}^{\prime}$, with the boundary conditions

$$
\begin{aligned}
& \lim _{r^{\prime} \rightarrow \infty} \boldsymbol{v}^{\prime}=\boldsymbol{v}_{\infty}, \\
& \forall \boldsymbol{n}^{\prime}, \boldsymbol{v}^{\prime} \cdot \boldsymbol{n}^{\prime}=\dot{R}, \text { at }\left|\boldsymbol{x}^{\prime}\right|=R,
\end{aligned}
$$

where $1=\nabla^{\prime} \varphi$ denotes the velocity field as seen from the comoving system, and $r^{\prime}=\left|\boldsymbol{x}^{\prime}\right|$. It is straightforward to check that the solution to that problem is given by

$\varphi\left(\boldsymbol{x}^{\prime}\right)=\frac{1}{2} \frac{R^{3}}{r^{\prime 2}} \boldsymbol{v}_{\infty} \cdot \boldsymbol{n}^{\prime}-\frac{\dot{R} R^{2}}{r^{\prime}}+\boldsymbol{v}_{\infty} \cdot \boldsymbol{r}^{\prime}$ 

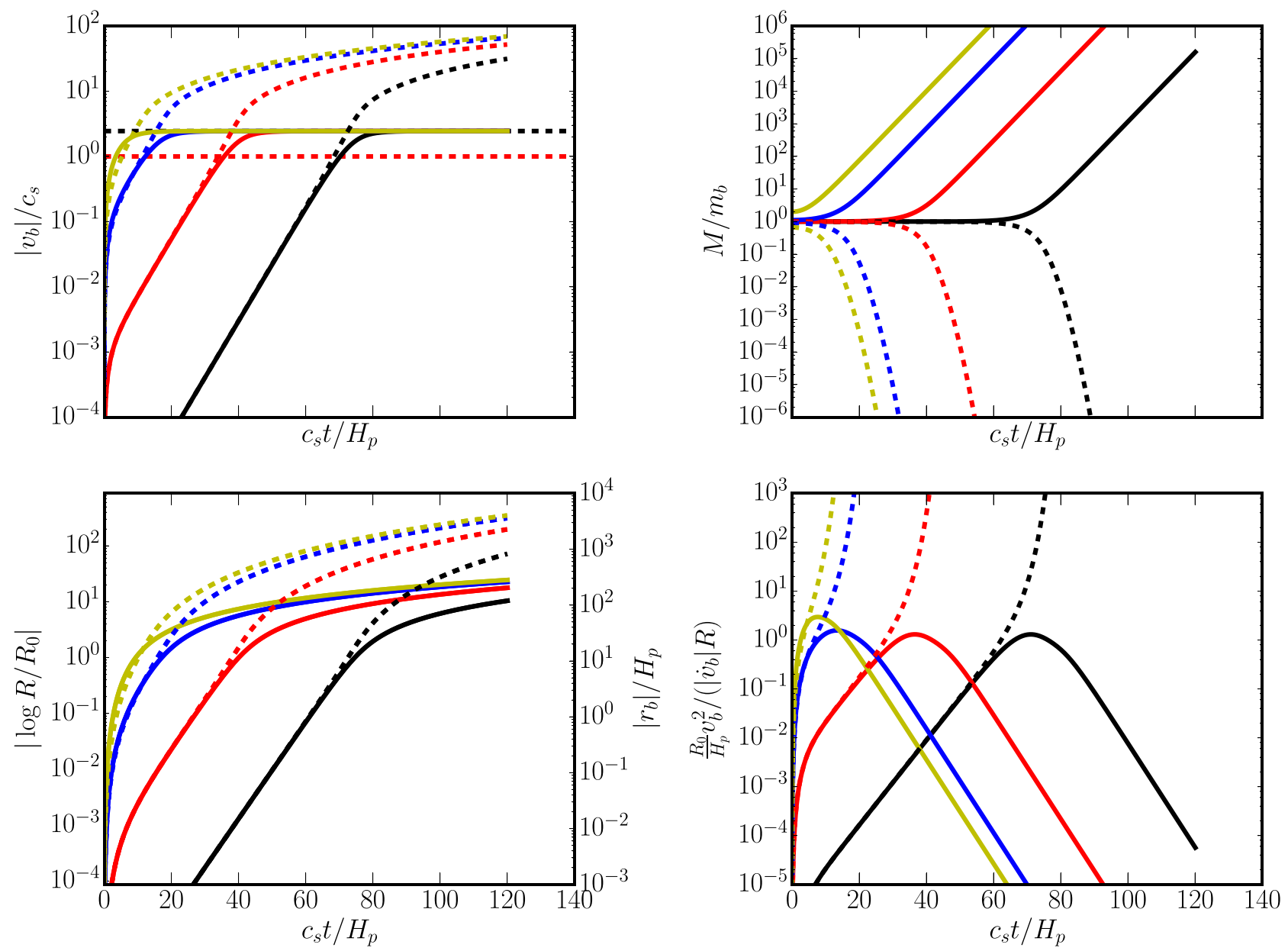

Figure 1. Solution of the bubble motion for $\Delta \nabla=10^{-1}$ and different initial bubble density perturbations (different colours correspond to different magnitude of the perturbation). Upper left panel: evolution of the bubble's velocity $\left(\left|v_{\mathrm{b}}(t)\right|\right)$. Upper right panel: evolution of $\omega=M / m_{\mathrm{b}}$. Bottom left panel: evolution of the bubble's expansion and position. Bottom right panel: evolution of the ratio $v_{\mathrm{b}}{ }^{2} /\left(\dot{\mathrm{v}}_{\mathrm{b}} R\right)$. The dashed lines correspond to the cases where $\delta \rho>0$, for which $v_{\mathrm{b}}<0, r_{\mathrm{b}}<0$, and $\log R / R_{0}<0$. The red horizontal dashed line in the left upper panel shows the (conservative) limit $v_{\mathrm{b}}=c_{\mathrm{S}}$ above which the theory is not valid. The black horizontal dashed line in the left panels is the asymptotic velocity $v_{\mathrm{b}}^{\infty}=\sqrt{6} \sim 2.45$ (see the text).

This is an extension of the solutions discussed in sections 10 and 11 of Landau \& Lifshitz (1987) in the case of an expanding sphere. Computing the derivatives, we obtain

$\boldsymbol{v}^{\prime}=-\frac{3 R^{3}}{2 r^{\prime 3}} \boldsymbol{n}^{\prime}\left(\boldsymbol{v}_{\infty} \cdot \boldsymbol{n}^{\prime}\right)+\boldsymbol{n}^{\prime} \frac{\dot{R} R^{2}}{r^{\prime 2}}+\frac{1}{2} \frac{R^{3}}{r^{\prime 3}} \boldsymbol{v}_{\infty}+\boldsymbol{v}_{\infty}$

The velocity field as seen from the system in which the bubble is in movement with velocity $v_{\mathrm{b}}$ can be obtained from a direct galilean transformation:

$v(x)=v^{\prime}\left(x^{\prime}\right)+v_{\mathrm{b}}=v^{\prime}\left(x-r_{\mathrm{b}}\right)+v_{\mathrm{b}}$.

Using $\boldsymbol{v}_{\infty}=-\boldsymbol{v}_{\mathrm{b}}$, we find,

$\boldsymbol{v}=\frac{3 R^{3}}{2 r^{\prime 3}} \boldsymbol{n}^{\prime}\left(\boldsymbol{v}_{\mathrm{b}} \cdot \boldsymbol{n}^{\prime}\right)+\boldsymbol{n}^{\prime} \frac{\dot{R} R^{2}}{r^{\prime 2}}-\frac{1}{2} \frac{R^{3}}{r^{\prime 3}} \boldsymbol{v}_{\mathrm{b}}$,

where it is worth noting that $r^{\prime}=\left|\boldsymbol{x}-\boldsymbol{r}_{\mathbf{b}}(t)\right|$ and $\boldsymbol{n}^{\prime}=(\boldsymbol{x}-$ $\left.\boldsymbol{r}_{\mathbf{b}}(t)\right) /\left|\boldsymbol{x}-\boldsymbol{r}_{\mathbf{b}}(t)\right|$ are functions of $\boldsymbol{x}$ and $t$. One can show that this velocity fields satisfies equations (14)-(16). This can be easily shown by noting that $\boldsymbol{x}^{\prime}=\boldsymbol{x}-\boldsymbol{r}_{\mathbf{b}}(t)$ implies that for any function $F(\boldsymbol{x})$, $\nabla^{\prime} F(\boldsymbol{x})=\nabla F(\boldsymbol{x}), \forall t$. The potential $\psi$ that produces the field $\boldsymbol{v}$ (equation 23) is given by

$\psi(\boldsymbol{x})=-\frac{1}{2} \frac{R^{3}}{r^{\prime 2}} \boldsymbol{v}_{\mathrm{b}} \cdot \boldsymbol{n}^{\prime}-\frac{\dot{R} R^{2}}{r^{\prime}}$.

\subsection{The instantaneous adjustment hypothesis}

In the following, we will assume that the shape of the velocity field instantaneously adjusts itself to the shape prescribed by equation (23) for the instantaneous values of $\boldsymbol{v}_{\mathrm{b}}(t), R(t)$ and $\dot{R}(t)$, i.e. we assume that

$\forall t, \boldsymbol{v}(\boldsymbol{x}, t)=\frac{3 R(t)^{3}}{2 r^{\prime 3}} \boldsymbol{n}^{\prime}\left(\boldsymbol{v}_{\mathrm{b}} \cdot \boldsymbol{n}^{\prime}\right)+\boldsymbol{n}^{\prime} \frac{\dot{R}(t) R(t)^{2}}{r^{\prime 2}}-\frac{1}{2} \frac{R(t)^{3}}{r^{\prime 3}} \boldsymbol{v}_{\mathrm{b}}$,

where the position of the bubble is given by $\boldsymbol{r}_{\mathbf{b}}(t)$ and $\boldsymbol{n}^{\prime}=\boldsymbol{x}^{\prime} /\left|\boldsymbol{x}^{\prime}\right|$, where $\boldsymbol{x}^{\prime}=\boldsymbol{x}-\boldsymbol{r}_{\mathrm{b}}(\mathbf{t})$ is the position as seen from the centre of the bubble. The velocity field of equation (25) fulfills the boundary conditions given by equations (15) and (16) at every time $t$. As $t$ and $\boldsymbol{x}$ are independent variables, it is easy to show that the potential $\psi(\boldsymbol{x}, t)$ that produces this field is

$\psi(\boldsymbol{x}, t)=-\frac{1}{2} \frac{R(t)^{3}}{r^{\prime 2}} \boldsymbol{v}_{\mathrm{b}} \cdot \boldsymbol{n}^{\prime}-\frac{\dot{R}(t) R(t)^{2}}{r^{\prime}}$.

In order for this hypothesis to hold, the fluid needs to adjust fast enough to the instantaneous velocity of the bubble. This hypothesis will hold if both the expansion velocity of the sphere and the translational velocity of the sphere are much smaller than the sound 

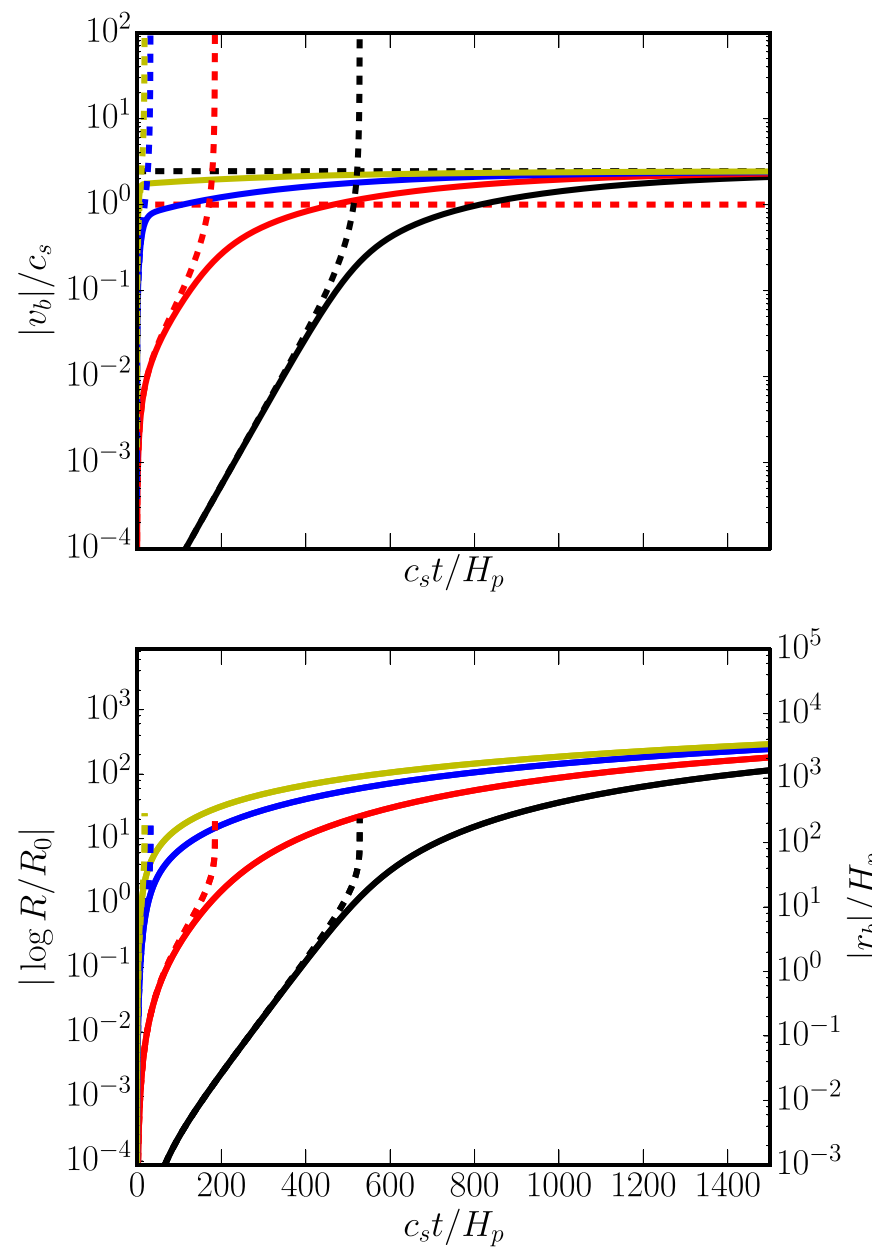
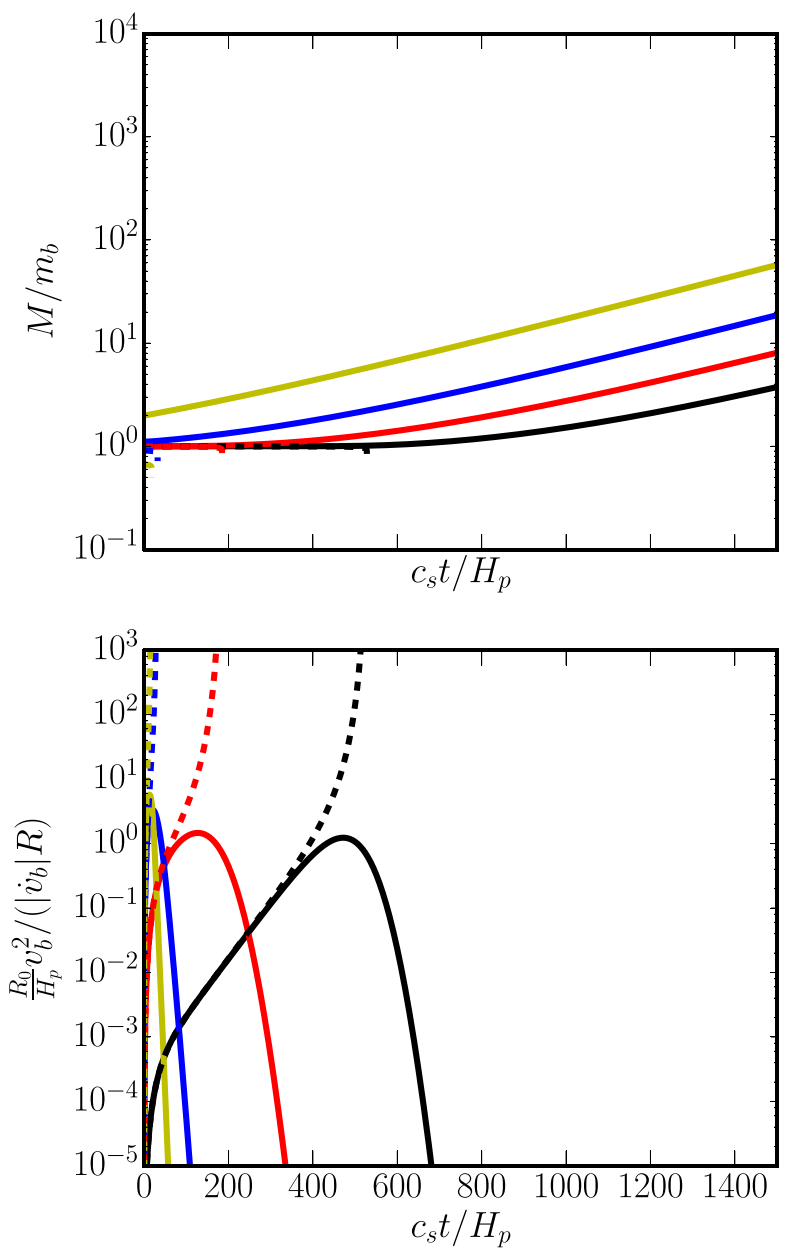

Figure 2. Same as Fig. 1 but for the case of $\Delta \nabla=10^{-3}$.

speed, i.e. if $v_{\mathrm{b}} \ll c_{\mathrm{s}}$ and $\dot{R} \ll c_{\mathrm{s}}$. In addition, we also assume that the time-scales related to the acceleration and the change in the expansion rate are small compared with the reaction time-scale of the fluid given by $\tau=R / c_{\mathrm{s}}-$ i.e. we assume that changes in $v_{\mathrm{b}}$ and $\dot{R}$ fulfill $\dot{R} / \ddot{R} \ll R / c_{\mathrm{s}}$ and $v_{\mathrm{b}} / \dot{v}_{\mathrm{b}} \ll R / c_{\mathrm{s}}$. Under the assumption of subsonic flows, this implies that $\left|\dot{\boldsymbol{v}}_{b}\right| \ll c_{\mathrm{s}}^{2} / R$ and $\ddot{R} \ll c_{\mathrm{s}}^{2} / R$. Note that the assumption of subsonic velocities is also compatible with the incompressibility approximation, which implies $c_{\mathrm{s}}=\infty$.

\subsection{Equation of motion for a moving and expanding sphere within a fluid at rest}

Once the velocity field is known, one can use this result to compute the force exerted by the fluid on the moving bubble by using Euler's equation

$\partial_{t} \boldsymbol{v}+(\boldsymbol{v} \cdot \nabla) \boldsymbol{v}=-\frac{\nabla P}{\rho}+\boldsymbol{g}$,

where $\boldsymbol{g}=-\nabla \Phi_{\mathrm{g}}$ is the gravitational acceleration. For an incompressible and irrotational fluid of constant density, equation (27) be written as

$\partial_{t} v+\nabla \frac{\boldsymbol{v}^{2}}{2}=-\nabla \frac{P}{\rho}-\nabla \Phi_{\mathrm{g}}$.
Equation (28) can then be rewritten, using $\nabla \psi=\boldsymbol{v}$, as

$\nabla\left(\partial_{t} \psi+\frac{\boldsymbol{v}^{2}}{2}+\frac{P}{\rho}+\Phi_{\mathrm{g}}\right)=0$.

Integrating this equation in space, we find

$\partial_{t} \psi+\frac{\boldsymbol{v}^{2}}{2}+\frac{P}{\rho}+\Phi_{\mathrm{g}}+c(t)=0$,

where $c(t)$ is a constant of integration. It can be obtained by noting that for $|\boldsymbol{x}| \rightarrow \infty$, the fluid is static $(\mathrm{v}=0)$ and in hydrostatic equilibrium $\left(\nabla\left(P / \rho+\Phi_{\mathrm{g}}\right)=0\right)$. This implies that ${ }^{6}$

$$
\left(\frac{P}{\rho}+\Phi_{\mathrm{g}}\right)_{|x| \rightarrow \infty}=C^{\prime},
$$

\footnotetext{
${ }^{6}$ Note that here the expression $|x| \rightarrow \infty$ means in fact at $\left|x-r_{\mathbf{b}}\right| \gg R$. Strictly speaking the limit $|x| \rightarrow \infty$ is ill-defined for a gravitational potential of a constant gravity field. Also, note that, as we are assuming that the hydrostatic pressure changes in much larger distances we are considering that at $\left|\boldsymbol{x}-\boldsymbol{r}_{\mathbf{b}}\right| \gg R$ the pressure $P_{\infty}$ depends on $z$ so that it can balance the changes in $\Phi_{\mathrm{g}}(z)$. Due that at the scales of the problem, $P_{\infty}$ remains almost constant, also $\Phi_{\mathrm{g}}$ must remain almost constant. In this context, it is useful to think the limit $|x| \rightarrow \infty$ on the $x y$-plane, where $\Phi_{\mathrm{g}}$ and $P_{\infty}$ are in fact strictly constant. Then the choice of $C^{\prime}=0$ corresponds to choosing $\Phi_{\mathrm{g}}=-g\left(z-r_{\mathrm{b}}\right)-P_{\infty}\left(z=r_{\mathrm{b}}\right) / \rho$.
} 
where $C^{\prime}$ is a constant that depends on the arbitrary choice of the definition of the gravitational potential. Noting that for $|\boldsymbol{x}| \rightarrow \infty$, we have that $\partial_{t} \psi \rightarrow 0$ and $v^{2} \rightarrow 0$, we see that equation (30) implies that $c(t)=-C^{\prime}$. For the sake of simplicity, we can set $C^{\prime}=c(t)=0$, and we obtain

$$
\frac{P}{\rho}=-\partial_{t} \psi-\frac{\boldsymbol{v}^{2}}{2}-\Phi_{\mathrm{g}} .
$$

The force $\boldsymbol{F}$ applied to the bubble is obtained by integrating equation (32) over the surface of the sphere $\partial V(t)$,

$$
\begin{aligned}
\boldsymbol{F} & =-\int_{\partial V} P \boldsymbol{n}^{\prime} \mathrm{d} S \\
& =\rho \int_{\partial V} \partial_{t} \psi \boldsymbol{n}^{\prime} \mathrm{d} S+\rho \int_{\partial V} \frac{\boldsymbol{v}^{2}}{2} \boldsymbol{n}^{\prime} \mathrm{d} S+\rho \int_{\partial V} \Phi_{g} \boldsymbol{n}^{\prime} \mathrm{d} S .
\end{aligned}
$$

The first integral in the right-hand side of equation (34) can be obtained using the definition of $\psi$, taking the time derivative $\partial_{t} \psi$ and evaluating over the sphere. We have

$$
\begin{aligned}
\partial_{t} \psi= & -\frac{3}{2} \dot{R}\left(\boldsymbol{v}_{\mathrm{b}} \cdot \boldsymbol{n}^{\prime}\right)-\frac{R}{2}\left(\dot{\boldsymbol{v}}_{\mathbf{b}} \cdot \boldsymbol{n}^{\prime}\right) \\
& -\ddot{R} R-2 \dot{R}^{2}, \text { for }\left|\boldsymbol{x}-\boldsymbol{r}_{\mathbf{b}}(\boldsymbol{t})\right|=R .
\end{aligned}
$$

Integrating over the whole sphere, we obtain

$\int_{\partial V}\left(-\partial_{t} \psi\right) \boldsymbol{n}^{\prime} \mathrm{d} S=2 \pi R^{2} \dot{R} \boldsymbol{v}_{\mathrm{b}}+\frac{2 \pi}{3} R^{3} \dot{\boldsymbol{v}}_{\mathrm{b}}$.

The second integral in the right-hand side of equation (34) can be directly computed once the velocity field is evaluated over the surface of the sphere:

$\boldsymbol{v}(\boldsymbol{x})=\left(v_{\mathrm{b}} \cos \theta+\dot{R}\right) \boldsymbol{n}^{\prime}+v_{\mathrm{b}} \frac{\sin \theta}{2} \mathbf{e}_{\theta}$, at $\left|\boldsymbol{x}-\boldsymbol{r}_{\mathrm{b}}(\boldsymbol{t})\right|=R(t)$,

where we have defined the spherical coordinates $r^{\prime}, \theta$ (zenithal angle) and $\phi$ (azimuthal angle) measured from the instantaneous centre of the sphere, and $\boldsymbol{e}_{\theta}$ is the unitary vector in the azimuthal direction. From equation (37), we obtain

$v(\boldsymbol{x})^{2}=\left(v_{\mathrm{b}} \cos \theta+\dot{R}\right)^{2}+\left(v_{\mathrm{b}} \frac{\sin \theta}{2}\right)^{2}, \quad$ at $\left|\boldsymbol{x}-\boldsymbol{r}_{\mathrm{b}}(\boldsymbol{t})\right|=R(t)$.

Integrating over the whole sphere, we obtain

$\int_{\partial V} \frac{v^{2}}{2} \boldsymbol{n}^{\prime} \mathrm{d} S=\frac{4 \pi}{3} \dot{R} R^{2} \boldsymbol{v}_{\mathrm{b}}$

where we have used that $\boldsymbol{e}_{z}=\boldsymbol{e}_{z^{\prime}}$ and $\boldsymbol{v}_{\mathrm{b}}=v_{\mathrm{b}} \boldsymbol{e}_{z}$.

Finally, the last integral in the right-hand side of equation (34) can be integrated using that

$\int_{\partial V} \Phi_{\mathrm{g}} \boldsymbol{n}^{\prime} \mathrm{d} S=\int_{V} \nabla \Phi_{\mathrm{g}} \mathrm{d} V=-\boldsymbol{g} V(t)$,

where $V(t)=4 \pi R(t)^{3} / 3$ is the volume of the expanding sphere.

Using equations (36), (38), and (40) in equation (34), the force exerted by the fluid on the moving bubble is

$$
\boldsymbol{F}=-\int_{\partial V} P \boldsymbol{n}^{\prime} \mathrm{d} S=-\frac{4 \pi R^{3}}{3} \rho \boldsymbol{g}-\frac{2 \pi}{3} \rho R^{2} \dot{R} \boldsymbol{v}_{\mathrm{b}}-\frac{2 \pi}{3} \rho R^{3} \dot{\boldsymbol{v}}_{\mathrm{b}} .
$$

\subsection{The acceleration of the bubble}

The equation of motion for the moving sphere, under all the previously mentioned assumptions, is

$m_{\mathrm{b}} \dot{\boldsymbol{v}}_{\mathbf{b}}=-\int_{\partial V} P \boldsymbol{n}^{\prime} \mathrm{d} S+m_{\mathrm{b}} \boldsymbol{g}$,

where $m_{\mathrm{b}}$ is the mass of the bubble $\left(m_{\mathrm{b}}=4 \pi R^{3} \rho_{\mathrm{b}} / 3\right)$, and the pressure integral is given by 41 . Using the definition $M=4 \pi R^{3} \rho / 3$ (i.e. the mass of a bubble of same radius but with the density of the fluid), equation (42) gives a very simple expression for the acceleration of the bubble;

$\dot{\boldsymbol{v}}_{\mathrm{b}}=\frac{\left(m_{\mathrm{b}}-M\right)}{\left(m_{\mathrm{b}}+M / 2\right)} \boldsymbol{g}-\frac{M}{2\left(m_{\mathrm{b}}+M / 2\right)} \frac{\dot{R}}{R} \boldsymbol{v}_{\mathrm{b}}$.

This is the correct version of the acceleration derived by Pasetto et al. (2014) in their equation (24). The first thing that is apparent from the first term in equation (43) is that, in the regime corresponding to our physical approximations, the acceleration of a bubble at rest is smaller by a factor $1+M /\left(2 m_{\mathrm{b}}\right)$ compared with the Archimedes principle for a static fluid. While this might be surprising at first glance, its physical explanation is quite simple. Within the approximation of equation (25), the fluid is forced to be accelerated when the bubble is accelerated. By looking at the stagnation points on top and below the bubble, it becomes clear that the fluid there moves at every time at the same velocity as the bubble. In order to fulfill Euler's equation for a velocity field that changes with time, some forces must be exerted at the boundary of the fluid (and equivalently, its reaction felt on the moving bubble). Consequently, the factor $1+M /\left(2 m_{\mathrm{b}}\right)$ accounts for the fact that, in order to accelerate, and fulfill equation (25), the bubble must carry the nearby fluid with it. The force exerted on the bubble by the surrounding medium is also responsible for the second term in equation (43). In this case, the term arises from the fact that, as the bubble expands, more fluid needs to be accelerated to fulfill equation (25). This term acts in the same orientation as the velocity, but its direction is determined by the sign of $\dot{R}$. Depending on whether the bubble is expanding or contracting, this term acts in the same direction as the velocity $v_{\mathrm{b}}$ or in the opposite one. In the latter case, it acts as a drag. It is worth noting that the claim of Pasetto et al. (2014) that this drag-like term reconciles the potential flow approximation with d'Alembert paradox is wrong, as this force is only present in the case of contracting or expanding spheres, and it is in no way related to real drag forces, which can be of viscous or turbulent origin. This is apparent from the fact that the force acts in the opposite direction, than that of a real drag force, in the case of contracting bubbles. Also, it is easy to see from equation (21) that the relative velocity of the fluid and the sphere has a tangential component at the surface of the sphere, contrary to what is known to happen at boundary layers.

Equation (43) has been derived under the assumption that the flow remains irrotational (potential) at all times. This is a very strong physical assumption and it would be necessary to investigate to which extent this will be an appropriate description of a given real fluid. For a compressible, viscous fluid moving under a conservative body force, we have that the vorticity $(\nabla \times \boldsymbol{v})$ fulfills

$$
\begin{aligned}
\frac{\mathrm{D}(\nabla \times \boldsymbol{v})}{\mathrm{D} t}= & ((\nabla \times \boldsymbol{v}) \cdot \nabla) \boldsymbol{v}-(\nabla \times \boldsymbol{v})(\nabla \cdot \boldsymbol{v}) \\
& +\nabla \times\left(\frac{\nabla \cdot \tau}{\rho}\right)+\frac{\nabla \rho \times \nabla P}{\rho^{2}},
\end{aligned}
$$

where $\mathrm{D} / \mathrm{D} t$ denotes the Lagrangian derivative and $\tau$ is the viscous stress tensor. In the general case, density will depend both on 
temperature and pressure. This implies that, in most cases, $\nabla \rho \times \nabla P$ $\neq 0$. Even if the flow is irrotational at the beginning of motion, one should expect that vorticity $(\nabla \times \boldsymbol{v})$ will be created at later times in a real flow by the last term in the right-hand side of equation (44). In addition, the absence of a drag force in equation (43) reminds us of the existence of boundary layers in real fluids around solid bodies, where viscosity cannot be completely neglected. In boundary layers, the third term in the right-hand side of equation (44) will also lead to the creation of vorticity. Consequently, even if the initial condition is that of an irrotational flow, there is no reason to expect that the flow will remain irrotational at all times. Besides the hypotheses done on the flow, the derivation of equation (43) also assumes that the bubble remains spherical at all times. However, equation (32) shows that pressure differences at the surface of the bubble should deform it as soon as it starts to move, unless internal forces prevent it (e.g. in a solid body). Because of all these assumptions, the use of equation (43) to describe the movement of spherical bubbles in stellar interiors might not be valid unless proven otherwise for each particular case.

Finally, up to now we have not made any assumption on the properties of the 'bubble' element. However, in a convection theory, we want the bubble to be made of the same material as the surrounding fluid. In the next section, we adopt an equation of state for the fluid inside the sphere and use it to describe the dynamics of the bubble.

\section{MOTION OF AN ISOLATED BUBBLE - SOLUTIONS AND ASYMPTOTIC BEHAVIOURS}

\subsection{General case}

While it is not our aim in this paper to develop a convection theory, we want to assess the expected behaviour for the motion of the bubble under the equation of motion derived in the previous section. The projected equation of motion of the bubble in the radial direction is

$\dot{v}_{\mathrm{b}}=-\frac{m_{\mathrm{b}}-M}{m_{\mathrm{b}}+\frac{M}{2}} g-\frac{1}{2} \frac{\dot{R}}{R} \frac{M}{m_{\mathrm{b}}+\frac{M}{2}} v_{\mathrm{b}}$,

with $M=4 \pi / 3 R^{3} \rho$ the buoyant mass, and $m_{\mathrm{b}}$ the bubble mass.

To solve the bubble motion through the whole convective region, we apply equation (45) at a given location of a stellar stratification. This is the spirit of solving a problem using local approach: the force balance that determines the acceleration of the bubble is computed in a local approach, and the result is used to determine the motion of the bubble through the convective region. This means that we need to specify the value of the thermodynamic variables, $T, \rho$ and $P$, as well as their stratification given by $H_{\mathrm{p}}, H_{\rho}$ and $\nabla=\mathrm{d} \log T / \mathrm{d} \log P$. Only four of them can be independently set, as they are related by the equation of state $\rho(T, P)$ of the stellar material, which implies

$\frac{\mathrm{d} \rho}{\rho}=\alpha \frac{\mathrm{d} P}{P}-\delta \frac{\mathrm{d} T}{T}$,

and consequently

$\nabla=\frac{\alpha}{\delta}-\frac{1}{\delta} \frac{H_{\mathrm{p}}}{H_{\rho}}$,

where $\alpha=(\partial \log \rho / \partial \log P)_{T}$ and $\delta=-(\partial \log \rho / \partial \log T)_{P}$. In order to solve equation (45), we need to know the evolution of $R$ and $M$ as the bubble evolves.
The evolution of the buoyant mass $M$ can be easily obtained by taking the time derivative of its definition:

$\frac{\dot{M}}{M}=3 \frac{\dot{R}}{R}+\frac{\dot{\rho}}{\rho}$,

since $\dot{\rho}=\mathrm{d} \rho(r(t)) / \mathrm{d} t=-\rho v_{\mathrm{b}} / H_{\rho}$, we have

$\frac{\dot{M}}{M}=3 \frac{\dot{R}}{R}-\frac{v_{\mathrm{b}}}{H_{\rho}}$.

The evolution of the radius $R$ of the bubble can be obtained from the equation of state (equation 46) and the assumption of subsonic motions. From equation (46), it is immediate that

$\frac{\dot{\rho}_{\mathrm{b}}}{\rho_{\mathrm{b}}}=\alpha \frac{\dot{P}}{P}-\delta \frac{\dot{T}_{\mathrm{b}}}{T_{\mathrm{b}}}$,

where we label with $b$ the thermodynamic quantities inside the bubble, and we have used that $P_{\mathrm{b}}=P(r(t))$. Using the fact that the mass of the bubble is constant, i.e. $\dot{\rho}_{\mathrm{b}} / \rho_{\mathrm{b}}=-3 \dot{R} / R$, and using equation (3), we finally get that the expansion of the bubble is governed by

$\frac{\dot{R}}{R}=\frac{\delta}{3} \frac{\dot{T}_{\mathrm{b}}}{T_{\mathrm{b}}}+\frac{\alpha}{3} \frac{v_{\mathrm{b}}}{H_{\mathrm{p}}}$.

To solve the dynamics, it is still necessary to know the evolution of the temperature of the bubble $T_{\mathrm{b}}$. This cannot be derived without taking into account the amount of heat lost (or gained) by the bubble as it moves. The energy balance of the bubble is given by (see Kippenhahn, Weigert \& Weiss 2012),

$\frac{\mathrm{d} q}{\mathrm{~d} t}=c_{P} \frac{\mathrm{d} T}{\mathrm{~d} t}-\frac{\delta}{\rho} \frac{\mathrm{d} P}{\mathrm{~d} t}$.

The heat flux $\boldsymbol{F}$ from the bubble is given by

$\boldsymbol{F}=-k_{\mathrm{rad}} \nabla T$, where $k_{\mathrm{rad}}=\frac{4 a c}{3} \frac{T_{\mathrm{b}}{ }^{3}}{\kappa_{\mathrm{b}} \rho_{\mathrm{b}}}$.

Estimating that the temperature gradient between the bubble and the surrounding fluid is $\mathrm{d} T / \mathrm{d} R \simeq\left(T(r)-T_{\mathrm{b}}\right) / R$, the heat losses from the spherical bubble are given by

$\frac{\mathrm{d} q}{\mathrm{~d} t} \simeq \frac{3}{\rho_{\mathrm{b}} R^{2}} k_{\mathrm{rad}}\left(T(r)-T_{\mathrm{b}}\right)$.

Replacing equation (54) in equation (52) gives

$\frac{\dot{T}_{\mathrm{b}}}{T_{\mathrm{b}}} \simeq \frac{3 k_{\mathrm{rad}}}{\rho_{\mathrm{b}} R^{2} c_{P}}\left[\frac{T(r)}{T_{\mathrm{b}}}-1\right]-\nabla_{\mathrm{ad}} \frac{v_{\mathrm{b}}}{H_{\mathrm{p}}}$,

where in the second term of the right-hand side, we replaced $\dot{P}=$ $-P v_{\mathrm{b}} / H_{\mathrm{p}}$, and used that $\nabla_{\mathrm{ad}}=(P \delta) /\left(c_{P} \rho_{\mathrm{b}} T_{\mathrm{b}}\right)$.

Equations (45), (49), (51) and (55), together with the stratification of the star $P(r), \rho(r), T(r), H_{\mathrm{p}}(r) H_{\rho}(r)$ and $\nabla(r)$, allow to solve the motion of the bubble. The reader should also be aware, however, that in order to use equation (45) to describe the motion of a bubble in a real flow, one should first show that the flow remains irrotational at all times. This is not trivial and in principle there is no reason to state that the generation of vorticity will be small. Equations (45), (49), (51) and (55), show that, even within the picture developed by Pasetto et al. (2014), it is necessary to take into account the radiative heat losses from the bubble (equation 55) before being able to solve the dynamics of the bubble. Equation (55) shows that depending on the typical time-scales for the expansion $\left(\tau_{\exp }=H_{\mathrm{p}} / v_{\mathrm{b}}\right)$ and thermal diffusion ( $\tau_{\mathrm{th}}=\rho_{\mathrm{b}} c_{P} R^{2} / 3 k_{\mathrm{rad}}$ ) the evolution of $T_{\mathrm{b}}$ will be completely different. In particular, as $\tau_{\text {th }} \propto R^{2}$, thermal diffusion always dominates the dynamics for bubbles that are small enough. 


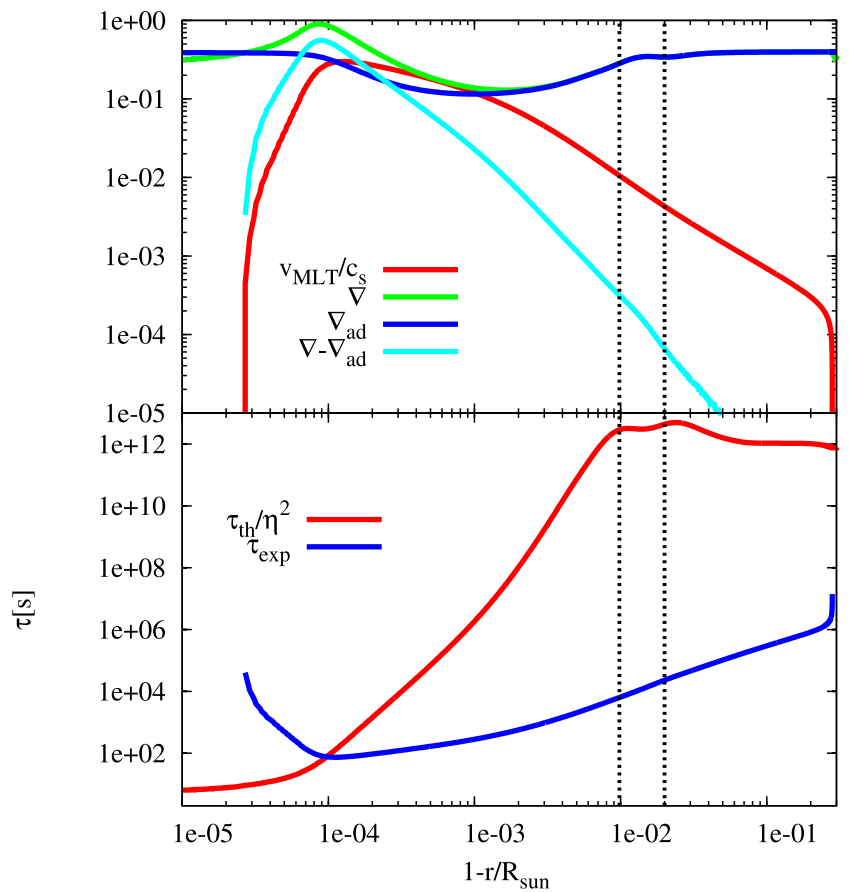

Figure 3. Properties of convective zone of the Garching solar model (GARSOM) as presented in Weiss \& Schlattl (2008), using the Grevesse \& Sauval (1998) solar composition, and updated nuclear reaction rates (Adelberger et al. 2011) and low-temperature Rosseland mean opacities (Ferguson et al. 2005). Upper panel: depth dependence of the convective velocity $v_{\mathrm{MLT}}$, actual temperature gradient $\nabla$, adiabatic gradient $\nabla_{\text {ad }}$ and superadiabaticity $\Delta \nabla=\nabla-\nabla_{\mathrm{ad}}$. Bottom panel: depth dependence of the thermal diffusion time-scale $\tau_{\text {th }}$ and the expansion time-scale $\tau_{\text {exp }}$ for convective elements of size $R=\eta H_{\mathrm{p}}$. The dotted vertical lines denote the layers at $r=0.98 \mathrm{R}_{\odot}$ and $\nabla_{\mathrm{ad}} \simeq 0.28310$ adopted by Pasetto et al. (2014) to compare their predictions with those of a solar calibrated MLT.

In the extreme case in which heat diffusion dominates, the bubble expands in isothermal equilibrium and there is no buoyancy. This is in stark contrast with the derivations performed by Pasetto et al. (2014) who solve (in their sections 4 and 5) the dynamics of the bubble without taking into consideration the role of heat diffusion. ${ }^{7}$ It is only in their section 6, after having solved the dynamics of the bubble, that they consider heat losses from the bubble. We will show in the next section that solving the dynamics without addressing the heat lost by the bubble can lead to extremely unphysical results.

In the bulk of the solar convective zone, one has $\tau_{\exp } \sim 10^{5}-10^{6} \mathrm{~s}$ and $\tau_{\text {th }} \sim 10^{12} \times \eta^{2}$ s for convective elements of size $R \sim \eta H_{\mathrm{p}}$ (see Fig. 3). The motion of convective elements in those cases is very close to adiabatic down to very small sizes - i.e. $\eta \gtrsim 10^{-3}$. Even in the very outer regions of the sun, one finds that the expansion timescale is shorter than the thermal time-scales, and the movement of a bubble is close to adiabatic for convective elements of size $R \sim H_{\mathrm{p}}$. For example, in the standard solar model of Fig. 3 (Weiss $\&$ Schlattl 2008), we see that at $r \simeq 0.999 \mathrm{R}_{\odot}$ one still finds that $\tau_{\text {exp }} \sim 10^{3} \mathrm{~s}$ and $\tau_{\text {th }} \sim 10^{6} \times \eta^{2} \mathrm{~s}$ and convective elements move almost adiabatically. While the assumption of adiabaticity is good to study the motion of convective elements in most of the solar

${ }^{7}$ In fact, the authors claim at the beginning of Section 4.2 that the dynamics of the bubble is solved under the assumption of adiabatic expansion. However, a careful examination of the derivations sheds that this hypothesis is never used. convective zone, one should keep in mind that it is in the regions far from adiabaticity that a better convection theory than MLT is needed to predict the correct value of the temperature gradient $\nabla$.

\subsection{Solutions for the adiabatic motion of the bubble}

It is well known that in the inner convective regions of stars the movement of convective elements of reasonable size is almost adiabatic due to the high density of the stellar matter. The assumption of adiabatic expansion greatly simplifies the treatment of equations (45), (49), (51) and (55). This allows for an easy test case for the dynamics of the bubble predicted by the method of Pasetto et al. (2014). For the sake of clarity, we will now consider the case of an ideal gas $(\alpha=\delta=1)$ with a constant adiabatic index $\gamma=\Gamma_{1}=\left(1-\nabla_{\mathrm{ad}}\right)^{-1}=5 / 3$. In the case of a bubble moving adiabatically in the stellar medium $\left(k_{\mathrm{rad}}=0\right)$ equation (55) can be directly substituted into equation (51) to give

$\frac{\dot{R}}{R}=\frac{v_{\mathrm{b}}}{3 H_{\mathrm{p}}}\left[1-\nabla_{\mathrm{ad}}\right]=\frac{v_{\mathrm{b}}}{3 \gamma H_{\mathrm{p}}}$.

Using equation (56) in equation (49), we can derive that

$\frac{\dot{M}}{M}=\frac{v_{\mathrm{b}}}{H_{\mathrm{p}}}\left[\nabla-\nabla_{\mathrm{ad}}\right]$,

where we have used the fact that $\nabla=1-H_{\mathrm{p}} / H_{\rho}$. The evolution of the bubble in the adiabatic case is given by the set of equations (45), (56) and (57). Note that equation (57) describes the usual Schwarzschild criterion. $M$ is the mass of the fluid that occupies the same volume as the bubble. If $M>m_{\mathrm{b}}$, the bubble will rise due to buoyancy, and if $M<m_{\mathrm{b}}$, the bubble will sink due to its own weight. Let us consider a bubble in equilibrium, i.e. $M=m_{\mathrm{b}}$, but under different values of $\Delta \nabla=\nabla-\nabla_{\text {ad }}$. When $\Delta \nabla>0$, a positive velocity perturbation will lead to an increase in $M$, leading to an upward force $\left(M>m_{\mathrm{b}}\right)$. On the other hand, a negative velocity perturbation will lead to a decrease of $M$ which will lead to a downward force $\left(M<m_{\mathrm{b}}\right)$. As expected, an unstable situation results. Similarly, $\Delta \nabla<0\left(\nabla<\nabla_{\text {ad }}\right)$ corresponds to a stable situation.

Substituting equation (56) in equation (45), we obtain the final set of equations that we need to solve:

$\dot{v}_{\mathrm{b}}=-\frac{m_{\mathrm{b}}-M}{m_{\mathrm{b}}+\frac{M}{2}} g-\frac{1}{6 \gamma H_{\mathrm{p}}} \frac{M}{m_{\mathrm{b}}+\frac{M}{2}} v_{\mathrm{b}}^{2}$,

$\frac{\dot{M}}{M}=\frac{v_{\mathrm{b}}}{H_{\mathrm{p}}} \Delta \nabla$.

It is best to formulate the system using non-dimensional quantities. We choose to normalize lengths with the pressure scaleheight $H_{\mathrm{p}}(=P / \rho g)$, velocities with the sound speed $c_{\mathrm{s}}(=\sqrt{\gamma P / \rho})$, and masses with the bubble mass $m_{\mathrm{b}}$. In these units, time is measured in units of $H_{\mathrm{p}} / c_{\mathrm{s}}$. The normalized system is

$\dot{v}_{\mathrm{b}}=-\frac{1}{\gamma} \frac{1-\omega}{1+\frac{\omega}{2}}-\frac{1}{6 \gamma} \frac{\omega}{1+\frac{\omega}{2}} v_{\mathrm{b}}^{2}$,
$\frac{\dot{\omega}}{\omega}=v_{\mathrm{b}} \Delta \nabla$.

where $\omega=M / m_{\mathrm{b}}$. Writing $m_{\mathrm{b}}=4 \pi / 3 R^{3} \rho_{\mathrm{b}}$, with $\rho_{\mathrm{b}}$ the bubble density, one has

$\omega(t)=\frac{\rho\left(r_{\mathrm{b}}(t)\right)}{\rho_{\mathrm{b}}(t)}$.

$\omega$ is the ratio between the background density and the bubble density. We define the density perturbation of the bubble as $\delta \rho=\rho_{\mathrm{b}}-\rho$, so that $\delta \rho / \rho=1 / \omega-1$. 
The system requires two initial conditions. The first initial condition is the initial velocity, $v_{\mathrm{b}}(t=0)$; the second initial condition is given by $\omega(t=0)=\rho\left(r_{\mathrm{b}}(t=0)\right) / \rho_{\mathrm{b}}(t=0)$, the initial density perturbation of the bubble. Having normalized lengths to the value of $H_{\mathrm{p}}$, the problem depends on one other parameter, the superadiabaticity $\Delta \nabla$.

It is worth noting that the radius of the bubble does not enter the adiabatic motion problem directly. However, once a solution $\left(v_{\mathrm{b}}(t)\right.$, $\omega(t))$ is known, the expansion of the bubble can be computed by integrating equation (56). In normalized form, it writes

$\frac{\dot{R}}{R}=\frac{v_{\mathrm{b}}}{3 \gamma}$.

We now rewrite it as

$\frac{\mathrm{d}}{\mathrm{d} t} \ln R=\frac{\dot{r}_{\mathrm{b}}}{3 \gamma}$,

which immediately leads to

$\ln \frac{R}{R_{0}}=\frac{r_{\mathrm{b}}}{3 \gamma}$

where $R_{0}$ is the bubble initial radius. The change in the bubble radius is directly related to the distance it has travelled from its initial position.

Equations (60) and (61) are solved numerically. As initial conditions, we consider that the bubble is at rest, $v_{\mathrm{b}}(t=0)=0$, and we use a density perturbation to initiate the motion of the bubble. We explore positive and negative initial density perturbations of different magnitudes, namely: $\delta \rho / \rho=-10^{-6},-10^{-3},-10^{-1},-0.5,10^{-6}$, $10^{-3}, 10^{-1}, 0.5-$ note that each $\delta \rho / \rho$ implies a different $\delta T_{\mathrm{b}} / T_{\mathrm{b}}$ so that pressure is balanced. We also investigate different values of the superadiabaticity, namely $\Delta \nabla=10^{-3}, 10^{-1}$. These values cover a range going from a nearly adiabatic stratification, as found in the deep stellar interior, to a value corresponding to a slight superadiabaticity, as found close to the stellar surface where the movement of the bubble can still be solved within the assumption of adiabatic expansion.

We show in Figs 1 and 2 the solutions of the bubble motion. When $\delta \rho<0$ (continuous lines), the bubble is rising and it reaches an asymptotic velocity, while $\omega=\rho / \rho_{\mathrm{b}}, r_{\mathrm{b}}$, and $\log \frac{R}{R_{0}}$ increases continuously with time. The value of the asymptotic velocity can be derived the following way. When $\omega \gg 1$, equation (60) becomes

$\dot{v}_{\mathrm{b}}=\frac{2}{\gamma}-\frac{1}{3 \gamma} v_{\mathrm{b}}^{2}$.

The asymptotic velocity corresponds to $\dot{v}_{\mathrm{b}}=0$, which leads to $v_{\mathrm{b}}^{\infty}=\sqrt{6}$. In physical units, this corresponds to $\sqrt{6} c_{\mathrm{s}}$. This value is shown as a horizontal dashed line in the left-hand panels of Fig. 1. The asymptotic velocity is supersonic, which is not consistent with the underlying assumptions of the theory. Therefore, it is clear that this asymptotic velocity cannot be used to compute a convective flux.

The time-scale on which the asymptotic velocity is reached depends only weakly on the magnitude of the initial density perturbation, but it depends strongly on the superadiabaticity. The smaller the superadiabaticity, the longer it takes to reach the asymptotic velocity. For the largest superadiabaticity explored here, $\Delta \nabla=10^{-1}$, the bubble expanded by a factor of $\sim 10$ and travelled a distance $\sim 10 H_{\mathrm{p}}$ when it reaches the asymptotic velocity. For $\Delta \nabla=10^{-3}$, the bubble expanded by a factor of $\sim 10-100$ and travelled over roughly $10^{3} H_{\mathrm{p}}$. For comparison, the number of pressure scaleheight in the entire Sun is roughly 30 . As a conclusion, it is clear that the time integration has to be stopped at some moment to make sure that the velocity of the bubble remains subsonic and that the bubble did not travel out of the convective region.

When $\delta \rho>0$ (dashed lines), the bubble sinks in the stratification. As a result, it contracts, and the magnitude of the velocity increases with time. We find that two different outcomes are obtained: the velocity diverges linearly in time for $\Delta \nabla=0.1$, and the velocity diverges at a finite time for $\Delta \nabla=10^{-3}$.

When the superadiabaticity is large enough $(\Delta \nabla=0.1$ in our case, see Fig. 1), $\omega=\rho / \rho_{\mathrm{b}}$ decreases rapidly as the bubble becomes more and more denser than its surrounding. When $\omega \ll 1$, equation (60) becomes

$\dot{v}_{\mathrm{b}}=-1 / \gamma$.

In physical units, this correspond to

$\dot{v}_{\mathrm{b}}=-g$.

As nothing in the theory prevents the bubble to stop contracting, its radius goes to zero and the bubble falls under the action of gravity alone (free-fall). Its velocity diverges, and it becomes rapidly supersonic.

When the superadiabaticy is small enough $\left(\Delta \nabla=10^{-3}\right.$ in our case, Fig. 2), $\omega=\rho / \rho_{\mathrm{b}}$ does not decrease quickly enough, and the increase in the velocity magnitude now results in the second term in equation (60) to be the dominant one. In this case, equation (60) can be written as

$\dot{v}_{\mathrm{b}}=-C v_{\mathrm{b}}^{2}$,

where $C$ is positive and can be considered constant in time. This gives immediately

$v_{\mathrm{b}}(t)=\frac{1}{-v_{\mathrm{b}}^{0}+C t}$,

where $v_{\mathrm{b}}^{0}$ is the (absolute) value of the bubble velocity at the moment where the buoyancy force becomes negligible. One sees from equation (70) that the bubble velocity diverges at $t=v_{\mathrm{b}}^{0} / C$. This is a remarkable result that at a first sight may look surprising, yet it can be understood in a very easy way and shows how unphysical the predictions from the theory are. In the extreme case of a bubble moving adiabatically in an adiabatic thermal stratification $(\Delta \nabla=0)$, the buoyancy mass and the density contrast remain constant. In this case, the first term in the acceleration equation remains constant while the second one increases as the bubble increases its speed. Once the second term becomes dominant, the bubble will contract extremely fast, shrinking to a point in a finite time-scale. Note that, as the density contrast remains constant to its initial value $\omega=\omega(t=0)$ this means that at each time the bubble has sunk deep enough so that its new density $\rho_{\mathrm{b}}(t)$ follows that of the background $\left(\rho\left(r_{\mathrm{b}}(t)\right)\right)$. In particular, this implies that when $R$ reaches $R=0$, the bubble has already sunk to an infinite depth.

A particularly interesting conclusion that arises from the solution of the motion of the adiabatic bubble is that there is no regime in which the acceleration of the bubble fulfills the key equation 41 of Pasetto et al. (2014). Not only $v_{\mathrm{b}}^{2} \neq-\dot{v}_{\mathrm{b}} R$, but, as shown in the bottom right panels of Figs 1 and 2 , the ratio $v_{\mathrm{b}}{ }^{2} /\left(\dot{v}_{\mathrm{b}} R\right)$ changes over orders of magnitude during the motion of the bubble. This is a very strong result as this approximation is key in the derivation of the convective flux in their work.

Finally, the previous results show that the theory cannot be used to describe the motion of the bubble at all times. The time integration has to be stopped when either one of the quantity $v_{\mathrm{b}}, R, r_{\mathrm{b}}$ reach a value where the underlying assumptions of the theory cannot be verified anymore. 


\section{DISCUSSION AND CONCLUDING REMARKS}

In the previous sections, we have addressed the theory of convection presented by Pasetto et al. (2014). As discussed in Section 2, their theory is both a local and a time-independent theory of convection, in the usual sense. In addition, we have shown that serious mathematical inconsistencies affect the derivation of the final equations in Pasetto et al. (2014), and that the key physical assumption of a rapidly expanding bubble $\left(v_{\mathrm{b}} / \dot{R} \ll 1\right)$ is in stark contradiction with the local and subsonic approach adopted by the authors which requires $R / H_{\mathrm{p}} \ll 1$. Yet, as we have shown in Sections 3 and 4 , it is possible to solve the dynamics of the bubble consistently under the main physical assumption of Pasetto et al. (2014), i.e. assuming a differentiated bubble moving in a potential flow. The detailed analysis of the resulting solutions for the evolution of the bubble show a very unphysical behaviour. This is not a surprise, as potential flows are known to be a far-fetched idealization of real fluids. Indeed, it is known since d'Alembert that potential flows predict zero drag, in strong contradiction with experience. This is the famous 'd'Alembert paradox' (le Rond d'Alembert 1768). Potential flows are popular in text books because they lead to analytically tractable problems. However, potential flows are rarely achieved in real day life, and they are mainly of academical interest (Feynman 1964). In fact, the d'Alembert paradox shows that the real flow around a body is not potential. Therefore, it is clear that the assumption of a potential flow has the drawback that the resulting theory will lack the drag that the fluid exerts on our bubble. Also, there is no physical reason to assume that a flow will remain potential even if that is the initial condition. The theory will necessarily be incomplete. In addition, it is worth noting that the relation between the acceleration, velocity and radius of the bubble derived by Pasetto et al. (2014), $v_{\mathrm{b}}^{2}=-\dot{v}_{\mathrm{b}} R$, does not exist in the detailed solution of the equations and is wrong by many orders of magnitude. This is important because this relation is used to derive the expression for the convective flux, which is key in their derivation of a convection theory.

All the previous points indicate that no accurate description of stellar convection can arise from the approach proposed by Pasetto et al. (2014). Yet, the authors claim that their theory is able to reproduce the solar predictions of a sun-calibrated MLT. While this claim looks surprising in view of the previous discussion, a closer inspection shows that there is no such agreement. In fact, in their table 1, the authors quote as a good agreement that their prediction for the temperature gradient $\nabla$ differs in only $1.7 \times 10^{-4}$ with the one predicted by the sun-calibrated MLT. While this difference might look small at first sight, it is a rather large discrepancy. The authors have chosen to compare their theory in a regime that is still very close to adiabatic convection - as can be seen from the fact that the convective flux is 6 orders of magnitude larger than the radiative one quoted in their table 1 . The relevant prediction for a convection theory is the degree of superadiabaticity $\Delta \nabla=\nabla-\nabla_{\mathrm{ad}}$. As seen in Fig 3, the superadiabaticity in that region of the solar convective zone is between $\Delta \nabla \sim 6 \times 10^{-5}$ and $3 \times 10^{-4}-$ either $^{8}$ at $r=0.98 \mathrm{R}_{\odot}$ or at the layer where $\nabla_{\mathrm{ad}} \sim 0.2831$. Therefore, the agreement for $\Delta \nabla$ between the theory of Pasetto et al. (2014)

\footnotetext{
${ }^{8}$ It should be noted that the 'solar model' adopted by Pasetto et al. (2014) is not a proper solar model, as the value of $\nabla$ at $R=0.98 \mathrm{R}_{\odot}$ is not the correct one, see Fig 3. Also, fig. 6 of Pasetto et al. (2014) shows the convective flux dominating down to $R=0.5 \mathrm{R}_{\odot}$, where the actual sun has no convective zone.
}

and the sun-calibrated MLT is not good, at best within an order of magnitude.

The study of the behaviour of the dynamics of the expanding bubble forces us to conclude that no improvement of stellar models can be expected from the approach presented by Pasetto et al. (2014). Indeed, the approach adopted by the authors is too simplistic, beside the inaccuracies discussed in Section 2, to be an accurate description of stellar convection. Since 20 years, numerical simulations of stellar convection have shown that the flow exhibits convective plumes, which are large-scale, coherent structures that emerge from the driving region and are able to propagate over significant distance before loosing their identities (Stein \& Nordlund 1989; Cattaneo et al. 1991; Brummell, Hurlburt \& Toomre 1996; Porter \& Woodward 2000; Brummell, Clune \& Toomre 2002; Viallet et al. 2013). The stratification has an important role in stellar convection, as it breaks the symmetry between upflows and downflows. For the case of stellar envelopes, where convection is driven by cooling at the photosphere, convective plumes propagate downwards, and are surrounded by a much broader and slower upflow. This is a result of mass conservation. Convective plumes are seen to maintain their coherence over long distances, i.e. larger than the pressure scaleheight, and they are responsible for the non-local character of convection. Furthermore, it is known from numerical simulations that convective plumes contribute to energy transport not only through the heat that they carry (enthalpy flux), but also through their kinetic energy. Due to the large Reynolds numbers that characterize stellar hydrodynamics, convective plumes induce shear instabilities as they propagate in the surrounding. As a result, they do not have a very definite surface, nor a definite shape, as they continuously mix with the surrounding. In some cases, this can reinforce the plume, as it entrain more mass and becomes stronger. In some other cases, it can lead to a destruction of the plume as it gets fully mixed with the surrounding, a phenomenon called 'detrainment' (Rieutord \& Zahn 1995; Rast 1998; Clyne et al. 2007). To be an improvement, future theories of stellar convection should take into account the non-local transport by convective plumes (Spruit 1997; Belkacem et al. 2006; Brandenburg 2015).

The picture adopted in Pasetto et al. (2014) ignores much of what has been learned from previous theoretical studies of stellar convection. Although the 'bubbles' which constitute the basis of Pasetto et al. (2014) theory could be at first be identified as representing convective plumes, it is clear that the picture adopted by the authors is too limited to really account for the observed properties of convective plumes:

(i) the authors adopt a local approach, in which the bubble size is restricted to length-scales smaller than the pressure scaleheight, and in which both the dynamics of the bubble, as well as the predicted temperature gradients, only depend on the properties at each stellar layer;

(ii) the authors assume that motions are subsonic. This a valid approximation for the deep interior, where convection is efficient. However, close to the photosphere, the Mach number can be very large so that the flow cannot be considered as subsonic. There, one has no other choice than to consider the fully compressible equations of hydrodynamics. For instance, at the photosphere the ram pressure of the fluid is large enough to modify hydrostatic equilibrium. This effect, which is described as due to a 'turbulent pressure', is neither taken into account in MLT nor in the approach of Pasetto et al. (2014);

(iii) the authors assume that the bubble has a well-delimited surface, along which the surrounding material is flowing. This picture 
is not able to account for shear instabilities that develop at the head of convective plumes, which of course have no definite surface. As mentioned previously, the way plumes entrain/detrain with the surrounding medium is key in setting their lifetime. This is the very reason why a mixing length is included ad hoc in the MLT picture.

Therefore, a non-local and time-dependent theory of stellar convection is still lacking, hampering progress in stellar physics. Better predictions for the structure of superadiabatic layers are required for asteroseismological studies. This requires to take into account compressibility (Mach numbers are of the order of one), and nonlocal effects due to plumes. State-of-the-art numerical simulations of photospheric convection are the most promising way to move beyond an MLT description of these layers. In the deep interior, although the thermal structure is know (the stratification is essentially adiabatic), a non-local theory of convection is needed to model the structure of the boundary layer between convective and stably stratified regions. This is timely, as both the extent and the efficiency of the mixing can now be probed with asteroseismology (Charpinet et al. 2011; Constantino et al. 2015). A theory of convection remains elusive as it is an outstanding challenge to capture the richness of the phenomenon into a mathematical description. Furthermore, the current stellar structure equations offer a too restricted framework to do better than MLT-like, local descriptions of convection. Progress in this challenging field will likely result from physical insight gained from numerical simulations, a complete re-thinking of the stellar evolution equations (e.g. with stellar evolution codes evolving towards mean-field hydrodynamics), and the use of the increasing quantity and quality of observational data available to constrain theoretical models. Unfortunately, the work by Pasetto et al. (2014) does not provide any useful foundation for the success of this challenging, but necessary, enterprise.

\section{ACKNOWLEDGEMENTS}

M3B is supported by a fellowship for postdoctoral researchers from the Alexander von Humboldt Foundation. This research was supported by the Munich Institute for Astro- and Particle Physics (MIAPP) of the DFG cluster of excellence 'Origin and Structure of the Universe'. Part of this work was funded by the European Research Council through grants ERC-AdG No. 341157-COCO2CASA.

\section{REFERENCES}

Adelberger E. G. et al., 2011, Rev. Mod. Phys., 83, 195

Arnett W. D., Meakin C., Viallet M., Campbell S. W., Lattanzio J. C., Mocák M., 2015, ApJ, 809, 30

Belkacem K., Samadi R., Goupil M. J., Kupka F., 2006, A\&A, 460, 173

Biermann L., 1932, Z. Astrophys., 5, 117

Brandenburg A., 2015, preprint (arXiv:1504.03189)

Brummell N. H., Hurlburt N. E., Toomre J., 1996, ApJ, 473, 494

Brummell N. H., Clune T. L., Toomre J., 2002, ApJ, 570, 825

Canuto V. M., 2011a, A\&A, 528, A76

Canuto V. M., 2011b, A\&A, 528, A77

Canuto V. M., 2011c, A\&A, 528, A78

Canuto V. M., 2011d, A\&A, 528, A79

Canuto V. M., 2011e, A\&A, 528, A80

Cattaneo F., Brummell N. H., Toomre J., Malagoli A., Hurlburt N. E., 1991, ApJ, 370, 282

Charpinet S. et al., 2011, A\&A, 530, A3

Clyne J., Mininni P., Norton A., Rast M., 2007, New. J. Phys., 9, 301

Constantino T., Campbell S. W., Christensen-Dalsgaard J., Lattanzio J. C., Stello D., 2015, MNRAS, 452, 123

de Bruijne J. H. J., 2012, Ap\&SS, 341, 31
Deng L., Xiong D. R., 2008, in Deng L., Chan K. L., eds, Proc. IAU Symp. 252, The Art of Modeling Stars in the 21st Century. Kluwer, Dordrecht, p. 83

Deng L., Xiong D. R., Chan K. L., 2006, ApJ, 643, 426

Ferguson J. W., Alexander D. R., Allard F., Barman T., Bodnarik J. G., Hauschildt P. H., Heffner-Wong A., Tamanai A., 2005, ApJ, 623, 585

Feynman R. P., 1964, in Leighton R. B., Sands M., eds, Feynman Lectures on Physics. Volume 2: Mainly Electromagnetism and Matter. AddisonWesley, Reading, MA

Flaskamp M., 2003, PhD thesis, Technische Univ. München

Grevesse N., Sauval A. J., 1998, Space Sci. Rev., 85, 161

Guzik J. A., Chaplin W. J., Handler G., Pigulski A., eds, 2014, Proc. IAU Symp. 301, Precision Asteroseismology. Cambridge Univ. Press, Universe

Kippenhahn R., Weigert A., Weiss A., 2012, Stellar Structure and Evolution. Springer-Verlag, Berlin Heidelberg

Kuhfuss R., 1986, A\&A, 160, 116

Kuhfuss R., 1987, PhD thesis, Technische Univ. München

Landau L. D., Lifshitz E. M., 1987, Fluid Mechanics, Second English Edition. Pergamon Press, Oxford

le Rond d'Alembert J., 1768, Paradoxe proposé aux géomètres sur la résistance des fluides. Opuscules mathématiques, vol. 5, Paris

Ludwig H.-G., Freytag B., Steffen M., 1999, A\&A, 346, 111

Magic Z., Weiss A., Asplund M., 2015, A\&A, 573, A89

Pasetto S., Chiosi C., Cropper M., Grebel E. K., 2014, MNRAS, 445, 3592

Porter D. H., Woodward P. R., 2000, ApJS, 127, 159

Prandtl L., 1925, Z. Angew. Math. Mech., 5, 136

Rast M. P., 1998, J. Fluid Mech., 369, 125

Rieutord M., Zahn J.-P., 1995, A\&A, 296, 127

Spruit H., 1997, Mem. Soc. Astron. Ital., 68, 397

Stein R. F., Nordlund A., 1989, ApJ, 342, L95

Trampedach R., Stein R. F., Christensen-Dalsgaard J., Nordlund Å., Asplund M., 2014, MNRAS, 445, 4366

Tremblay P.-E., Ludwig H.-G., Freytag B., Fontaine G., Steffen M., Brassard P., 2015, ApJ, 799, 142

Viallet M., Meakin C., Arnett D., Mocák M., 2013, ApJ, 769, 1

Viallet M., Meakin C., Prat V., Arnett D., 2015, A\&A, 580, A61

Vitense E., 1953, Z. Astrophys., 32, 135

Weiss A., Flaskamp M., 2007, in Kupka F., Roxburgh I., Chan K. L., eds, Proc. IAU Symp. 239, Convection in Astrophysics. Cambridge Univ. Press, Cambridge, p. 314

Weiss A., Schlattl H., 2008, Ap\&SS, 316, 99

Wuchterl G., Feuchtinger M. U., 1998, A\&A, 340, 419

Xiong D.-R., 1986, A\&A, 167, 239

\section{APPENDIX A: RELATIONSHIP BETWEEN $P_{\mathrm{b}}$ AND $P_{\infty}$}

It is also interesting to analyse how the predictions of equation (32) for the connection between the pressure inside the bubble $P_{\mathrm{b}}$ and the pressure of the fluid far away from the bubble $P_{\infty}$. For this some physical comment about the hypothesis of the spherical symmetry of the bubble is due. It is clear from equation (23) that the pressure on the surface of a sphere moving within a fluid is not constant. In the absence of any other forces, these differential forces will deform the shape of the bubble. Then, the hypothesis of a spherically symmetric bubble at all times is equivalent to assume that forces on the surface of the bubble are able to balance the differential forces and keep a spherical shape but do not prevent the sphere form expanding (i.e. a mechanical constraint).

In order to obtain the link between the pressure inside the bubble $P_{\mathrm{b}}$ (assumed to be filled with a homogeneous fluid) and the pressure in the fluid we can analyse the energy transferred during an adiabatic spherically symmetric expansion $\mathrm{d} V_{\mathrm{b}}$. Under the assumption that the surface forces only act to prevent the departure from spherical 
symmetry, we can then write that the work done by the sphere has to be equated by the energy received by the rest of the fluid, i.e.

$P_{\mathrm{b}} \mathrm{d} V_{\mathrm{b}}=-\int_{\partial V} P \boldsymbol{n}^{\prime} \cdot \mathrm{d} \boldsymbol{r}^{\prime} \mathrm{d} S$,

as $\mathrm{d} \boldsymbol{r}^{\prime}$ and $\boldsymbol{n}^{\prime}$ are parallel during and spherical expansion, we can then write

$P_{\mathrm{b}}=-\frac{\int_{\partial V} P \mathrm{~d} S}{4 \pi R^{2}}$.

Then, using equation (32), we find that

$P_{\mathrm{b}}=-\frac{\int_{\partial V}\left(\partial_{t} \psi+\frac{\mathrm{v}^{2}}{2}+\Phi_{\mathrm{g}}\right) \mathrm{d} S}{4 \pi R^{2}}$,

which provides a link between the pressure inside the bubble and the state of the surrounding material. Now we can make of of the choice of $C^{\prime}=0$ for the relation the between pressure and the gravitational potential far away from the sphere and write $\Phi_{\mathrm{g}}=$ $-g\left(z-r_{\mathrm{b}}\right)-P_{\infty}^{t} / \rho-$ where $P_{\infty}^{t}$ is the pressure far away from the bubble at the layer $z=r_{\mathrm{b}}(t)$.

$$
\begin{aligned}
P_{\mathrm{b}}= & -\frac{1}{4 \pi R^{2}}\left(\int_{\partial V} \partial_{t} \psi \mathrm{d} S+\int_{\partial V} \frac{\boldsymbol{v}^{2}}{2} \mathrm{~d} S+\right. \\
& \left.+\int_{\partial V}\left(-g z^{\prime}\right) \mathrm{d} S+\int_{\partial V} P_{\infty}^{t} \mathrm{~d} S\right) .
\end{aligned}
$$

Performing the integrations, we find that

$$
P_{\mathrm{b}}=P_{\infty}^{t}-\frac{\rho}{2} \dot{R}^{2}-\frac{\rho}{2} v_{\mathrm{b}}^{2} \frac{1}{4 \pi R^{2}} \int \frac{\sin ^{2} \theta}{2} \mathrm{~d} S-\ddot{R} R \rho-2 \rho \dot{R}^{2} .
$$

Using the definition of the adiabatic sound speed, we can now replace $\rho=\Gamma_{1} P_{\infty} / c_{\mathrm{s}}^{2}$ to get

$$
\begin{aligned}
P_{\mathrm{b}}= & P_{\infty}^{t}-\frac{\Gamma_{1} P_{\infty}}{c_{\mathrm{s}}{ }^{2}} \dot{R}^{2}-\frac{\Gamma_{1} P_{\infty}}{c_{\mathrm{s}}{ }^{2}} v_{\mathrm{b}}^{2} \frac{1}{4 \pi R^{2}} \int \frac{\sin ^{2} \theta}{2} \mathrm{~d} S \\
& -\frac{\Gamma_{1} P_{\infty}}{c_{\mathrm{s}}{ }^{2}} \ddot{R} R-\frac{\Gamma_{1} P_{\infty}}{c_{\mathrm{s}}{ }^{2}} 2 \dot{R}^{2} .
\end{aligned}
$$

Equation (A6) shows the fact that when $\dot{R} \ll c_{\mathrm{s}}, v_{\mathrm{b}} \ll c_{\mathrm{s}}$ and $\ddot{R} R \ll c_{\mathrm{s}}^{2}$, the pressure inside the bubble can be considered to be equal to that of the medium far away from the bubble $P_{\mathrm{b}} \simeq P_{\infty}^{t}$, as it is well known for subsonic flows.

This paper has been typeset from a $\mathrm{T}_{\mathrm{E}} \mathrm{X} / \mathrm{L} \mathrm{T}_{\mathrm{E}} \mathrm{X}$ file prepared by the author. 